\title{
Perfil epidemiológico de acidentes por animais peçonhentos em Minas Gerais
}

\author{
Epidemiological profile of accidents by venomous animals in Minas Gerais \\ Perfil epidemiológico de accidentes por animales venenosos en Minas Gerais
}

\section{Resumo}

Acidentes com animais peçonhentos são aqueles causados por animais capazes de produzir peçonha e inoculá-las. De acordo a Organização Mundial da Saúde (OMS), trata-se de um problema de saúde pública. O objetivo proposto foi descrever o perfil epidemiológico dos casos de acidentes por animais peçonhentos em Minas Gerais (MG), entre 2015 e 2017, evidenciando a espécie do animal que causou acidente, a faixa etária dos indivíduos acometidos, macrorregião de saúde por notificação e evolução dos casos. Estudo epidemiológico descritivo, retrospectivo com abordagem de natureza quantitativa. Os dados foram extraídos do SINAN, através de acesso ao Departamento de Informática do Sistema Único de Saúde (DATASUS). O levantamento de dados ocorreu no período de 1 a 30 de setembro de 2021. A população de interesse foram os casos notificados em MG no período entre 2015 a 2017. Os dados foram tabulados no 
Microsoft Office Excel® e tratados estatisticamente, por meio do programa Statistical Package for the Social Sciences (SPSS) versão 20.0. Por se tratar de um banco de domínio público, não foi necessário submeter o projeto ao Comitê de Ética em Pesquisa. Houve predominância da faixa etária de 20 a $59(58,8 \%)$, raça parda $(49,2 \%)$, sexo masculino $(54,8 \%)$, na macrorregião Norte $(18,8 \%)$, envolvendo escorpiões $(67,9 \%)$ e alto percentual cura $(97,1 \%)$. Assim, fazse necessário a elaboração estratégias para conter o quadro, tais como promoção de ações de educação em saúde para população sobre o assunto, a elaboração de protocolos operacionais padrão direcionados a prevenção e manejo de acidentes com animais peçonhentos.

Palavras-chave: Animais venenosos; Sistema de informação; Vigilância epidemiológica.

\begin{abstract}
Accidents with venomous animals are those caused by animals capable of producing venom and inoculating them. According to the World Health Organization (WHO), this is a public health problem. The proposed objective was to describe the epidemiological profile of cases of accidents by poisonous animals in Minas Gerais (MG), between 2015 and 2017, showing the species of animal that caused the accident, the age range of the affected individuals, health macro-region by notification and evolution of cases. Descriptive, retrospective epidemiological study with a quantitative approach. Data were extracted from SINAN, through access to the Information Technology Department of the Unified Health System (DATASUS). Data collection took place from September 1 to 30, 2021. The population of interest were the cases reported in MG in the period between 2015 and 2017. Data were tabulated in Microsoft Office Excel ${ }^{\circledR}$ and statistically treated using the program Statistical Package for the Social Sciences (SPSS) version 20.0. As it is a public domain bank, it was not necessary to submit the project to the Research Ethics Committee. There was a predominance of the age group from 20 to $59(58,8 \%)$, brown race $(49,2 \%)$, male $(54,8 \%)$, in the northern macro region $(18,8 \%)$, involving scorpions $(67,9 \%)$ and high cure percentage $(97,1 \%)$. Thus, it is necessary to develop strategies to contain the situation, such as promoting health education actions for the population on the subject, the development of standard operational protocols aimed at the prevention and management of accidents with venomous animals.
\end{abstract}

Keywords: Animals poisonous; Information systems; Epidemiological monitoring.

\title{
Resumen
}

Los accidentes con animales venenosos son los provocados por animales capaces de producir veneno e inocularlos. Según la Organización Mundial de la Salud (OMS), este es un problema de salud pública. El objetivo propuesto fue describir el perfil epidemiológico de los casos de accidentes por animales venenosos en Minas Gerais (MG), entre 2015 y 2017, mostrando la especie de animal que causó el accidente, el rango de edad de los individuos afectados, macrorregión de salud. por notificación y evolución de casos. Estudio epidemiológico descriptivo retrospectivo con abordaje cuantitativo. Los datos fueron extraídos del SINAN, a través del acceso al Departamento de Tecnología de la Información del Sistema Único de Salud (DATASUS). La recolección de datos se realizó del 1 al 30 de septiembre de 2021. La población de interés fueron los casos reportados en MG en el período entre 2015 y 2017. Los datos fueron tabulados en Microsoft Office Excel® y tratados estadísticamente mediante el programa Statistical Package for the Social Sciences (SPSS) versión 20.0. Al ser un banco de dominio público, no fue necesario presentar el proyecto al Comité de Ética en Investigación. Predominó el grupo de edad de 20 a 59 (58,8\%), raza morena (49,2\%), masculino $(54,8 \%)$, en la macro región norte $(18,8 \%)$, involucrando escorpiones $(67,9 \%)$ y alto porcentaje de curación. $(97,1 \%)$. Por ello, es necesario desarrollar estrategias para contener la situación, como la promoción de acciones de educación sanitaria para la población sobre el tema, el desarrollo de protocolos operativos estándar orientados a la prevención y manejo de accidentes con animales venenosos.

Palabras clave: Animales venenosos; Sistemas de información; Seguimiento epidemiológico.

\section{Introdução}

Acidentes com animais peçonhentos são aqueles causados por animais capazes de produzir peçonha e inocula-las, podendo apresentar dentes modificados, aguilhão, ferrão, quelíceras, cerdas urticantes, entre outros. De acordo a Organização Mundial da Saúde (OMS), são inúmeros os problemas de saúde pública e acomete, com maior frequência, populações mais vulneráveis que residem em condições precárias. No Brasil, devido ao alto número de notificações, esse agravo foi incluído na Lista de Notificação Compulsória, visando à adoção de estratégias, bem como ações que possibilitem a prevenção desse tipo de acidente (Vieira \& Machado, 2018).

O SINAN classifica os animais peçonhentos como aqueles que modificam ou produzem algum veneno, incluindo serpentes, escorpiões, aranhas, mariposas, abelhas, entre outros. No Brasil, através do SINAN, vinculado ao Departamento de Informática do Sistema Único de Saúde - DATASUS, foram registradas 1.352.976 notificações relativas a acidentes com animais peçonhentos no período de 2015 a 2017 (Brasil, 2019; Brasil, 2020). 
Além disso, evidenciou-se através dos estudos que, no período de 2015 a 2017, o estado de Minas Gerais (MG) notificou 102.808 casos de acidentes por animais peçonhentos, o que corresponde a aproximadamente $8 \%$ do total de casos no país, em que destes, mais de 100 mil casos envolveram acidentes com escorpião, na faixa etária 20 a 39 anos de idade e região norte do estado (Brasil, 2020).

Vale ressaltar que para se diagnosticar um acidente por animal peçonhento, é necessário identificar o animal que causou o evento, procedimento importante. Isso porque, na maioria dos casos, conduz a terapêutica necessária, pois permite a identificação de pacientes picados por serpentes não peçonhentas, auxiliando na indicação mais precisa do soro antiveneno, além de viabilizar o reconhecimento das espécies de importância médica. Contudo, quando não é possível identificar o animal causador, o tratamento é baseado no reconhecimento sintomático, com administração do soro antiveneno, específico para cada espécie e situação (Silva, 2017).

O atendimento e o tratamento são concedidos, gratuitamente e de forma integral, pelo Sistema Único de Saúde (SUS) que oferta atendimento de primeiros socorros em caso de acidentes com animais peçonhentos. Nesse sentido, reforça-se ainda que, não há recomendações da realização de torniquetes, garrotes, cortes, furos, expressão, queimadura ou sucção no local da lesão causada por animal peçonhento. Além disso, é vedada a aplicação de substâncias que possam causar infecções. A conduta recomendada é levar a vítima ao serviço de saúde mais próximo para receber tratamento adequado em tempo hábil (Almeida, 2020).

Assim, levando em consideração que os acidentes com animais peçonhentos são um problema de saúde pública e as publicações sobre esse tema são escassas, o presente artigo teve como objetivo descrever o perfil epidemiológico dos casos de acidentes por animais peçonhentos em MG, entre 2015 e 2017, evidenciando a espécie do animal que causou o acidente, a faixa etária dos indivíduos acometidos e a macrorregião de saúde por notificação, sendo que os dados levantados nesse estudo, poderão servir de subsídio teórico para pesquisas posteriores.

\section{Metodologia}

Tratou-se de um estudo epidemiológico descritivo, retrospectivo com abordagem de natureza quantitativa (Gil, 2002). Os dados foram extraídos do Sistema de Informação de Agravos de Notificação (SINAN), banco de dados obtido através do acesso ao Departamento de Informática do Sistema Único de Saúde (DATASUS) no endereço eletrônico (http://www.datasus.gov.br). O levantamento de dados ocorreu no período de 1 a 30 de setembro de 2021. Para evitar erros de retardo de notificação, optou-se por analisar os dados disponíveis até 2017, último ano em que constavam completos.

A população de interesse foram os habitantes do estado de $M G$ que sofreram acidentes por animais peçonhentos diagnosticados e registrados no período de 2015 a 2017, sendo analisados os seguintes parâmetros: faixa etária, macrorregião de saúde da notificação, o animal respectivo ao acidente e a evolução dos casos.

Os dados foram inicialmente tabulados no Microsoft Office Excel ${ }^{\circledR}$ e foram tratados estatisticamente (em termos de média, correlaç̃oes e números absolutos), por meio do programa Statistical Package for the Social Sciences (SPSS) versão 20.0. Por se tratar de um banco de domínio público, não foi necessário submeter o projeto ao Comitê de Ética em Pesquisa.

\section{Resultados e Discussão}

O presente estudo buscou descrever o perfil epidemiológico dos casos de acidentes por animais peçonhentos em MG, entre 2015 a 2017, evidenciando a espécie do animal que causou acidente, a faixa etária dos indivíduos acometidos e a macrorregião de saúde por notificação com intuito de entender a amplitude desta problemática. 
De acordo com a Tabela 1, os resultados encontrados a respeito das variáveis sociodemográficas demonstraram predominância de acidentes com animais peçonhentos no sexo masculino $(54,8 \%)$, dado este que se justifica pelo predomínio desse gênero atuando no principal meio laboral do Estado de Minas Gerais, a agropecuária (Meschial et al., 2013).

Ainda nesse contexto, houve predominância da faixa etária de 20 a $59(58,8 \%)$, dado este que corrobora com estudo de (Santos \& Souto, 2011), que indica os indivíduos mais ativos economicamente no estado e raça parda (49,2\%), que de acordo com último censo realizado pelo Instituto Brasileiro de Geografia e Estatística (IBGE) 2010, corresponde à maioria da população brasileira (Tabela 1). (Silva, 2015).

Tabela 1 - Características sociodemográficas (faixa etária, raça e sexo), em valores absolutos e percentual, da população acometida por acidentes com animais peçonhentos, por macrorregião de saúde do estado de MG, entre 2015 e 2017.

\begin{tabular}{lcccccccccc}
\hline \multicolumn{1}{c}{\begin{tabular}{l} 
Macrorregião de \\
\multicolumn{1}{c}{ Saúde }
\end{tabular}} & \multicolumn{3}{c}{ Idade } & & & & Raça & & & \multicolumn{2}{c}{ Sexo } \\
& & & & & & & & & \\
Norte & $20-59$ & 60 e+ & Branca & Preta & Amarela & Parda & Indígena & M & F \\
\cline { 2 - 10 } Centro & 5589 & 10751 & 3005 & 1828 & 963 & 106 & 15114 & 127 & 8863 & 10481 \\
Sul & 4566 & 10533 & 2468 & 3642 & 1416 & 151 & 9555 & 38 & 9921 & 8344 \\
Nordeste & 2648 & 7655 & 1905 & 8777 & 861 & 53 & 2238 & 22 & 7824 & 4384 \\
Leste & 2341 & 4251 & 1472 & 824 & 735 & 56 & 6628 & 118 & 4349 & 3715 \\
Triângulo do Norte & 1770 & 2938 & 915 & 763 & 423 & 90 & 2239 & 18 & 3006 & 2617 \\
Leste do Sul & 1540 & 4171 & 1388 & 3940 & 672 & 164 & 2176 & 13 & 3787 & 3315 \\
Triângulo do Sul & 1477 & 4270 & 1030 & 2410 & 788 & 109 & 3287 & 14 & 4116 & 2675 \\
Noroeste & 1461 & 3600 & 898 & 3446 & 464 & 66 & 1724 & 6 & 3284 & 1955 \\
Jequitinhonha & 1111 & 2805 & 650 & 1650 & 302 & 45 & 2339 & 14 & 2612 & 1955 \\
& 854 & 1736 & 434 & 508 & 306 & 49 & 1891 & 8 & 1678 & 1349 \\
Sudeste & & & & & & & & & & \\
Oeste & 756 & 2374 & 611 & 1693 & 428 & 51 & 639 & 8 & 2426 & 1315 \\
Vale do Aço & 741 & 2349 & 617 & 1912 & 265 & 36 & 1105 & 3 & 2221 & 1488 \\
Centro Sul & 706 & 1520 & 454 & 865 & 205 & 39 & 1124 & 3 & 1542 & 1139 \\
Total absoluto & 468 & 1246 & 390 & 1229 & 207 & 23 & 731 & 3 & $/ 1427$ & 858 \\
Total em \% & 26028 & 60379 & 16237 & 33487 & 8035 & 1038 & 50541 & 395 & 56356 & 46295 \\
\hline & $25,3 \%$ & $58,8 \%$ & $15,8 \%$ & $32,6 \%$ & $7,8 \%$ & $1 \%$ & $49,2 \%$ & $0,3 \%$ & $54,8 \%$ & $45 \%$ \\
\hline
\end{tabular}

Fonte: Ministério da Saúde/SVS - Sistema de Informação de Agravos de Notificação - Sinan Net.

A análise dos resultados cadastrados no SINAN acerca da ocorrência de acidentes por animais peçonhentos por regiões de MG, demonstraram que das 102.659 ocorrências notificadas no período de 2015 a 2017 na macrorregião Norte obteve maior índice de ocorrência (18,8\%), seguida das regiões Centro (17,1\%), Sul (11,8\%), Nordeste (7,8\%), Triângulo do Norte $(6,9 \%)$, Leste do Sul (6,6\%), Triângulo do sul (5,8\%), Leste $(5,4 \%)$, Noroeste $(4,4 \%)$, Sudeste $(3,6 \%)$, Oeste $(3,6 \%)$, Jequitinhonha $(2,9 \%)$, Vale do Aço (2,6\%) e Centro Sul (2,2\%), como demonstrado na Tabela 2. 
Tabela 2- Ocorrência de acidentes por animais peçonhentos por regiões de MG, entre 2015 e 2017.

\begin{tabular}{lccccc}
\hline \multicolumn{1}{c}{ MACRORREGIÃO DE SAÚDE } & & ANO & & \multicolumn{2}{c}{ Total em } \\
& $\mathbf{2 0 1 5}$ & $\mathbf{2 0 1 6}$ & $\mathbf{2 0 1 7}$ & TOTAL & $\%$ \\
\hline Norte & 5970 & 5864 & 7514 & 19348 & $18,8 \%$ \\
Centro & 5260 & 5551 & 6757 & 17568 & $17,1 \%$ \\
Sul & 3492 & 4054 & 4662 & 12208 & $11,8 \%$ \\
Nordeste & 2307 & 2281 & 3476 & 8064 & $7,8 \%$ \\
Triângulo do norte & 2065 & 2279 & 2758 & 7102 & $6,9 \%$ \\
Leste do sul & 2052 & 2142 & 2585 & 6779 & $6,6 \%$ \\
Triângulo do sul & 1864 & 1780 & 2315 & 5959 & $5,8 \%$ \\
Leste & 1647 & 1753 & 2223 & 5623 & $5,4 \%$ \\
Noroeste & 1295 & 1386 & 1886 & 4567 & $4,4 \%$ \\
Sudeste & 1104 & 1170 & 1467 & 3741 & $3,6 \%$ \\
Oeste & 1056 & 1182 & 1471 & 3709 & $3,6 \%$ \\
Jequitinhonha & 870 & 967 & 1187 & 3024 & $2,9 \%$ \\
Vale do aço & 765 & 731 & 1186 & 2682 & $2,6 \%$ \\
Centro sul & 622 & 761 & 902 & 2285 & $2,2 \%$ \\
Total & 30369 & 31901 & 40389 & 102659 & $100 \%$ \\
\hline
\end{tabular}

Fonte: Ministério da Saúde/SVS - Sistema de Informação de Agravos de Notificação - Sinan Net.

De acordo com estudos analisados, essa evidência está relacionada com a concentração de fatores que influenciam a alta incidência de casos na macrorregião Norte, tais como a crescente urbanização e o desmatamento devido atividade agropecuária intensa da região, fatores que alteram o habitat natural da fauna e flora existentes, justificando o crescente número de incidentes nessa macrorregião (Alencar, Araújo \& Carvalho, 2019).

Somado a isto tem-se o baixo índice de desenvolvimento humano (IDH), em comparação a outras macrorregiões de MG, característica que contribui para o agravamento dos casos ocorridos na macrorregião Norte. Isso porque reflete uma população com menor índice de educação em saúde e assim, menor preparo para lidar com manejo correto dos acidentes envolvendo animais peçonhentos (Gonçalves et al., 2020).

Ainda na Tabela 2, observou-se elevação expressiva dos casos com o passar dos anos, sobretudo entre os anos 2016 e 2017, com aumento dos casos, representando aproximadamente 30\% de acréscimo. Esse aumento na frequência de acidentes pode estar relacionado a variáveis como clima, fauna, flora e índice de desmatamento, que determinam a frequência desses incidentes, como já relatado quando mostrado a maior ocorrência de acidentes na região norte (Alencar et al., 2019).

Na tabela 3, verificou-se que 67,3\% dos acidentes por animais peçonhentos envolveram escorpiões no período analisado (2015 a 2017). Esse achado relaciona-se com o habitat natural desses animais, que são prevalentes, sobretudo, em regiões quentes e úmidas, características comuns no estado de MG. Contudo, devido às mudanças climáticas associadas à degradação ambiental associada ao desequilíbrio ecológico, é possível identificar a ocorrência de acidentes envolvendo escorpiões em locais antes atípicos, sobretudo devido à migração desses animais para centros urbanos, o que ocasiona maior frequência de casos nessas regiões. Além disso, a ausência de saneamento básico e infraestrutura favorece sua disseminação (Ferreira \& Rocha, 2019; Silveira \& Machado, 2017). 
Tabela 3 - Ocorrência de acidentes por animais peçonhentos, por ano e agente agressor, em MG, entre 2015 e 2017.

\begin{tabular}{lccccc} 
Tipo de Acidente & $\mathbf{2 0 1 5}$ & $\mathbf{2 0 1 6}$ & $\mathbf{2 0 1 7}$ & Total & Total em \% \\
Escorpiões & 19924 & 21568 & 27986 & 69478 & $67,3 \%$ \\
Aranhas & 3306 & 3424 & 3673 & 10403 & $10 \%$ \\
Serpentes & 2618 & 2511 & 3306 & 8435 & $8,1 \%$ \\
Abelhas & 2310 & 2255 & 2611 & 7176 & $6,9 \%$ \\
Outros & 1422 & 1263 & 1575 & 4260 & $4,1 \%$ \\
Lagartas lonomia & 738 & 819 & 1096 & 2653 & $2,5 \%$ \\
Ignorado & 196 & 222 & 338 & 756 & $0,7 \%$ \\
Total geral & & & 103161 & $100 \%$ \\
\hline
\end{tabular}

Fonte: Ministério da Saúde/SVS - Sistema de Informação de Agravos de Notificação - Sinan Net.

Em 2017, houve um aumento expressivo de casos (26,5\%) em relação ao ano anterior (2016), sendo o período com maior número de acidentes, com predomínio dos casos escorpiônicos (67,3\%). Esse dado, pode ser correlacionado com as alterações climáticas e a ampliação do desmatamento no estado de MG, relatado pelo Instituto Nacional de Meteorologia (INMET), o que contribuiu para o surgimento de novos casos pelas condições favoráveis para a proliferação dos escorpiões, principalmente na área urbana, visto que o deslocamento desses animais aumenta diante desses fatores. Correlaciona-se esse achado com o aumento do número de notificações que ocorreu no Brasil neste mesmo período como demonstrado na tabela 3 (Souza, Pinto \& Feitosa, 2017).

Em relação a ocorrência de acidentes por peçonha e macrorregião, observou-se na tabela 4, maior prevalência geral concentra-se macrorregião Norte (18,8\%), seguida da Centro (17,1\%), Sul (11,8\%) Nordeste (7,8\%), Triângulo do Norte (6,9\%), Leste do Sul (6,6\%), Triângulo do sul (5,8\%), Leste (5,4\%), Noroeste (4,4\%), Sudeste e Oeste (3,6\%), Jequitinhonha (2,9\%), Vale do Aço $(2,6 \%)$ e Centro Sul (2,2\%). Conforme os dados, em relação ao animal mais prevalente nestes acidentes, observouse um padrão em ambas as regiões, sendo encontrada prevalência no número de acidentes por escorpiões (67,4\%), seguido por aracnídeos (10\%), serpentes $(8,1 \%)$, lagartas lonomia $(2,5 \%)$ e abelhas $(6,95 \%)$.

Nesse contexto, a alta incidência de acidentes envolvendo aracnídeos nas regiões Sul e Centro-sul do estado de MG pode ser justificada pela localização geográfica das mesorregiões citadas, as quais ficam próximas da Mata Atlântica e do cerrado brasileiro, biomas que sofreram graves danos ao longo dos anos devido ações antrópicas (Moraes, A. R. da Silva , E.R. da Silva, Coelho \& Pardal, 2021).

Além da degradação ambiental, a infraestrutura sanitária deficiente no entorno das cidades propicia a busca desses artrópodes por novos habitats, sendo eles entulhos, lixos, construções ou até mesmo o ambiente doméstico, de modo que encontram abrigo e alimento. Dessa forma, o contato com seres humanos é mais frequente, tornando os acidentes recorrentes. Exemplo disso foi evidenciado em estudo realizado na cidade de Juiz de Fora, situada no sudeste de MG, a qual é bem próxima ao bioma tropical, considerada endêmica para a prevalência de artrópodes, sobretudo araneísmo (Martins, Andrade, R.C.P.A. Vieira, A.A.P. Vieira \& Raposo, 2011).

Nas demais regiões, é perceptível a prevalência por acidentes escorpiônicos, o que corrobora com o estudo realizado por (Gonçalves et al. 2020), que demonstrou a relação entre o desequilíbrio ecológico gerado pelas mudanças promovidas pelo homem, como o desmatamento e a urbanização e aumento da população de escorpiões e sua adaptação às habitações urbanas. Além disso, os índices socioeconômicos contribuem de forma direta para a alta prevalência dos escorpiões, pois de acordo com estudos analisados, a maioria dos incidentes ocorrem em habitações precárias, sem infraestrutura e saneamento básico. 
Tabela 4 - Ocorrência de acidentes por agente agressor e macrorregião de saúde de MG, entre 2015 e 2017.

\begin{tabular}{|c|c|c|c|c|c|c|c|c|c|}
\hline $\begin{array}{l}\text { Macrorregião de } \\
\text { Saúde }\end{array}$ & Serpentes & Aracnídeos & Escorpiões & $\begin{array}{l}\text { Lagartas } \\
\text { lonomia }\end{array}$ & Abelhas & Outros & Ignorados & Total & $\begin{array}{l}\text { Total } \\
\text { em \% }\end{array}$ \\
\hline Norte & 649 & 373 & 16841 & 97 & 661 & 572 & 155 & 19348 & $18,8 \%$ \\
\hline Centro & 956 & 1909 & 11222 & 922 & 1415 & 941 & 203 & 17568 & $17,1 \%$ \\
\hline Sul & 952 & 3359 & 3341 & 865 & 2469 & 1111 & 111 & 12208 & $11,8 \%$ \\
\hline Nordeste & 576 & 147 & 7141 & 23 & 105 & 58 & 14 & 8064 & $7,8 \%$ \\
\hline Triangulo do Norte & 611 & 297 & 5479 & 58 & 397 & 229 & 31 & 7102 & $6,9 \%$ \\
\hline Leste do Sul & 880 & 1014 & 4538 & 38 & 145 & 135 & 29 & 6779 & $6,6 \%$ \\
\hline Triangulo do Sul & 430 & 269 & 4643 & 64 & 364 & 169 & 20 & 5959 & $5,8 \%$ \\
\hline Leste & 429 & 174 & 4697 & 13 & 202 & 97 & 11 & 5623 & $5,4 \%$ \\
\hline Noroeste & 593 & 276 & 3016 & 68 & 410 & 193 & 11 & 4567 & $4,4 \%$ \\
\hline Sudeste & 725 & 902 & 1777 & 59 & 117 & 133 & 28 & 3741 & $3,6 \%$ \\
\hline Oeste & 422 & 345 & 2234 & 98 & 362 & 202 & 46 & 3709 & $3,6 \%$ \\
\hline Jequitinhonha & 259 & 232 & 2200 & 94 & 99 & 89 & 51 & 3024 & $2,9 \%$ \\
\hline Vale do aço & 497 & 277 & 1821 & 19 & 43 & 22 & 3 & 2682 & $2,6 \%$ \\
\hline Centro sul & 348 & 741 & 326 & 226 & 341 & 276 & 27 & 2285 & $2,2 \%$ \\
\hline Total & 8327 & 10315 & 69276 & 2644 & 7130 & 4227 & 740 & 102659 & $100 \%$ \\
\hline
\end{tabular}

Fonte: Ministério da Saúde/SVS - Sistema de Informação de Agravos de Notificação - Sinan Net.

Na evolução de casos por agente agressor observou-se um alto índice de cura (97,19\%). A análise dos dados evidenciou ainda uma maior mortalidade por escorpião, dado o alto número de acidentes escorpiônicos. É relevante ainda o grande número proporcional de óbitos causados por abelhas $(3,3 \%)$ se comparado com serpentino $(2,2 \%)$, aracnídeo $(1,4 \%)$, escorpiônico $(1,1 \%)$ e de lagartas (0,0003\%), apesar de em números absolutos ser um dos menores como demonstrado na Tabela 5.

Tabela 5 - Evolução de casos por agente agressor, em MG, entre 2015 e 2017.

\begin{tabular}{|c|c|c|c|c|c|c|c|c|c|}
\hline $\begin{array}{c}\text { Evolução } \\
\text { caso }\end{array}$ & Ignorado & Serpentes & Aranhas & Escorpiões & Lagartas & Abelhas & Outros & Total & $\begin{array}{l}\text { Total } \\
\text { em \% }\end{array}$ \\
\hline Ignorado & 65 & 450 & 310 & 1609 & 55 & 142 & 100 & 2731 & $2,6 \%$ \\
\hline Cura & 691 & 7961 & 10076 & 67784 & 2597 & 7005 & 4154 & 100268 & $97,1 \%$ \\
\hline $\begin{array}{c}\text { Óbito pelo } \\
\text { agravo } \\
\text { notificado }\end{array}$ & - & 18 & 15 & 79 & 1 & 24 & 6 & 143 & $0,1 \%$ \\
\hline $\begin{array}{c}\text { Óbito por } \\
\text { outra } \\
\text { causa }\end{array}$ & - & 6 & 2 & 6 & - & 5 & - & 19 & $0,01 \%$ \\
\hline Total & 756 & 8435 & 10403 & 69478 & 2653 & 7176 & 4260 & 103161 & $100 \%$ \\
\hline
\end{tabular}


O elevado percentual de cura pode estar relacionado ao fato do Brasil ser autossuficiente na produção de antivenenos. A disponibilização e repasse desses soros, é responsabilidade do Ministério da Saúde, na figura das Secretarias de Saúde, de acordo com a incidência de casos de cada região, o que depende da notificação de casos ao SINAN e dos investimentos do Governo nos Institutos produtores desses antídotos (Vaz, Brazil \& Paixão, 2020). O planejamento e amplitude da distribuição nacional dos soros é possibilitada por meio da atualização dos Sistemas de Informação, tal como o SINAN, o que interfere diretamente no percentual de cura, visto que este é relacionado à disponibilidade e acesso ao soro (Salomão, Luna \& Machado, 2018).

Nesse sentido, a alta taxa de cura constatada relaciona-se com a capacidade brasileira em produzir soros específicos contra tais animais peçonhentos. O país é referência mundial nesse aspecto por possuir institutos especializados em medicina tropical como o Butantan em São Paulo, Fundação Ezequiel Dias em MG e Instituto Vital Brasil no Rio de Janeiro. Colabora também a distribuição a nível nacional do Ministério da Saúde que verifica a necessidade de cada localidade conforme o registro no SINAN (Vance et al., 2017).

\section{Conclusão}

Esta pesquisa descreveu o perfil epidemiológico dos casos de acidentes por animais peçonhentos ocorridos no estado de Minas Gerais (MG) no período de 2015 a 2017, contribuindo como referência para outras publicações na área. Além disso, servirá como subsídio teórico para implementação de intervenções relacionadas à prevenção.

Baseado nos dados coletados foi possível descrever o perfil epidemiológico de acidentes por animais peçonhentos, em que se evidenciou que a maioria dos acidentes ocorrem com indivíduos do sexo masculino, pardos, em idade funcional (faixa etária de 20 a 59 anos), com trabalho laboral na zona rural, na macrorregião Norte de Minas Gerais, sendo escorpiões os principais envolvidos com alto percentual de evolução para cura após o tratamento.

Os achados demonstraram que os acidentes com animais peçonhentos ainda são um problema de saúde pública, sobretudo no estado de Minas Gerais. Nesse contexto, faz-se necessário a elaboração estratégias para conter o quadro, tais como promoção de ações de educação em saúde para população sobre o assunto, a elaboração de protocolos operacionais padrão direcionados especificamente a prevenção e manejo de acidentes com animais peçonhentos de acordo com o perfil epidemiológico do local.

Assim, acidentes com animais peçonhentos são evitáveis quando se prioriza condutas preventivas, portanto, estados e municípios devem estar aptos a implementar medidas que evitem esses agravos por meio da execução e planejamentos pelos órgãos sanitários.

Ademais, com os resultados demonstrados fica evidente que são necessários futuros trabalhos investigativos em relação à epidemiologia desses agravos, uma vez que é uma temática de interesse público.

\section{Referências}

Alencar, E.S., Araújo, M. H. S. \& Carvalho, A. V .(2019). Acidentes por animais peçonhentos no município de Guaraí (TO) no período de 2015-2017. Medicus, $1(1), 10-21$.

Almeida, C.B. (2020). Acidentes por animais peçonhentos no estado do Amapá em 2019. Braz. J. of Develop, 6(12), 103538-50.

Barbosa, I.R., Nunes, A. D. S. Amador, A. E (2017). Araneísmo no município de Natal, Rio Grande do Norte no período de 2007 a 2014 . Revista Ciência Plural,

3(1), 22-34.

https://www.researchgate.net/publication/340254116_ARANEISMO_NO_MUNICIPIO_DE_NATAL_RIO_GRANDE_DO_NORTE_NO_PERIODO_DE_2 007_A_2014

Ministério da Saúde. (2021, Outubro 25). TabNet Win32 3.0: ACIDENTE POR ANIMAIS PEÇONHENTOS - Notificações registradas no Sistema de Informação de Agravos de Notificação - Minas Gerais. Datasus. http://tabnet.datasus.gov.br/cgi/tabcgi.exe?sinannet/cnv/animaismg.def

SINAN. (08 de Março de 2016). Portal SINAN. Fonte: Site do SINAN: http://portalsinan.saude.gov.br/acidente-por-animais-peconhentos 
Instituto Brasileiro de Geografia e Estatística. (2010, Novembro 04). Censo Demográfico: População residente por cor ou raça e religião, tabela 2094. Sidra IBGE.https://sidra.ibge.gov.br/tabela/2094\#/n1/all/n2/all/n3/all/v/1000093/p/last\%201/c86/allxt/c133/0/d/v1000093\%201/1/v,p+c86,t+c133/resultado

NOTA TÉCNICA 004/17. (2017). Estação Chuvosa em Minas Gerais. Belo Horizonte, Instituto Nacional de Meteorologia. https://portal.inmet.gov.br/uploads/notastecnicas/Nota_tecnica_CEDEC_04_17.pdf

Ferreira, L. C. Rocha, Y. C. S (2019). Incidência de acidentes por escorpiões no município de Januária, Minas Gerais, Brasil. Journal Health NPEPS, 4(1), 228241. doi:0.30681/252610103351

GIL, Antônio Carlos (2002) - Como elaborar projetos de pesquisa - 4. Ed. - São Paulo; Atlas

Gonçalves, J.E., Medeiros, S.M.F.R.S., Cavalcanti, I.D.L., Mendes, R.C.M.G., Bezerra, I. N. M, \& Nóbrega, M.M. (2020). Acidentes por animais peçonhentos: uma análise do perfil epidemiológico da região Nordeste do Brasil no período de 2010 a 2019. Pesquisa, Sociedade e Desenvolvimento, 9(10), 10.33448

Martins, F. J., Andrade, N.S., Vieira, R.C.P.A. Vieira, A.A.P., \& Raposo, N.R.B. (2011). Perfil dos acidentes causados por aranhas na área de abrangência sanitária do município de Juiz de Fora - MG. Rev APS. 14(3): 303-312. https://periodicos.ufjf.br/index.php/aps/article/view/14825/7900

Campo Meschial, W., Ferreira Martins, B., dos Reis, L.M., da Silva Lira Ballani, T., Lopes Barboza, C., \& Félix de Oliveira, M.L., (2013). Internações hospitalares de vítimas de acidentes por animais peçonhentos. Rev Rene, 14(2), 311-319. https://www.redalyc.org/articulo.oa?id=324027986009

Moraes, F.C.A. de., Silva, A.R. da., Silva, E. R. da., Coelho, J. de S., \& Pardal, P.P. de O. (2021). Relação dos biomas nos acidentes peçonhentos no Brasil/ Relationship of biomes in venomous accidents in Brazil/ Relación de biomas en accidentes venenosos en Brasil. Journal Health NPEPS, 6(1). Recuperado de https://periodicos.unemat.br/index.php/jhnpeps/article/view/5320

da Silva Vaz, V. H., Vital Brazil, O. A., \& Almeida Paixão, A. E. (2020). Propriedade intelectual do soro antiofídico: a efetividade a partir da correlação entre os investimentos do governo federal nos principais institutos responsáveis pela produção do soro e realização de pesquisas para o tratamento de acidentes ofídicos no Brasil, com relação ao número de vítimas fatais dos acidentes. Cadernos Saúde Coletiva [online], v. 28, n. 3, pp. 409-421. https://doi.org/10.1590/1414462X202028030018

Ribeiro dos Santos, G., \& Gomes dos Santos Souto, K. (2014, Abril, 14). O desenvolvimento no norte de minas na perspectiva da sudene. Revista Desenvolvimento Social, 12(2), 69-78. Recuperado de https://www.periodicos.unimontes.br/index.php/rds/article/view/1970

Salomão, M. G., Luna, K. P. O., \& Machado, C. (2018). Epidemiologia dos acidentes por animais peçonhentos e a distribuição de soros: estado de arte e a situação mundial. Rev. Salud Pública. 20 (4), 523-529. doi: 10.15446/rsap.v20n4.70432

Silva, A. M. da., Bernarde, P. S., \& Abreu, L. C. de. (2015). Acidentes com animais peçonhentos no Brasil por sexo e idade. Journal of Human Growth and Development, 25(1), 54-62.http://pepsic.bvsalud.org/scielo.php?pid=S0104-12822015000100007\&script=sci_abstract

Silva, J. H. J., Giansante, S., Ribeiro da Silva, R. C., Barbosa da Silva, G., Benedito Silva, L., \& Castelo Branco Pinheiro, L. (2017). Perfil epidemiológico dos acidentes com animais peçonhentos em Tangará da Serra, Mato Grosso, Brasil (2007-2016) / Epidemiological profile of accidents with peconving animals in Tangará de Serra-MT, Brazil (2007-2016) / Perfil epidemiológico de los. Journal Health NPEPS, 2(1), 5-15. https://periodicos.unemat.br/index.php/jhnpeps/article/view/179

Silveira, J.L., \& Machado, C. (2017). Epidemiologia dos acidentes por animais peçonhentos nos municípios do sul de Minas Gerais. J Health NPEPS, 2 (Supl1), 88-101. https://periodicos.unemat.br/index.php/jhnpeps/article/view/1774/1655

Souza, L.M., Pinto, R.N.L., Feitosa, D.T., \& Jr, N.J.S. (2017). Estudo retrospectivo do escorpionismo no Estado de Goiás (2003-2012). Estud Vida Saúde, 44, 100-114.https://www.researchgate.net/publication/321580860_Retrospective_study_in_scorpionism_Goias_State_2003-2012

Vance, C., Allan Santos, C. M., Bueno F., Armada, F., Giffoni, M., Camelo M., Ospina M. L., Mondragón N. F., \& Gadelha de Abreu, R. (2017). Produção de antivenenos: experiências e desafios do Brasil e Colômbia: Interferência da indústria de alimentos na saúde coletiva? Saúde ao Sul: ISAGS/UNASUR, 10(1), 116. https://www.dndial.org/wp-content/uploads/2017/10/SaudeSur_ISAGS_B_Pecoul_Oct_2017.pdf

Vieira, G.P.S., \& MACHADO, C. (2018). Acidentes por animais peçonhentos na região serrana, Rio de Janeiro, Brasil. Journal Health NPEPS, 3(1),211227.doi: $10.30681 / 252610102776$ 